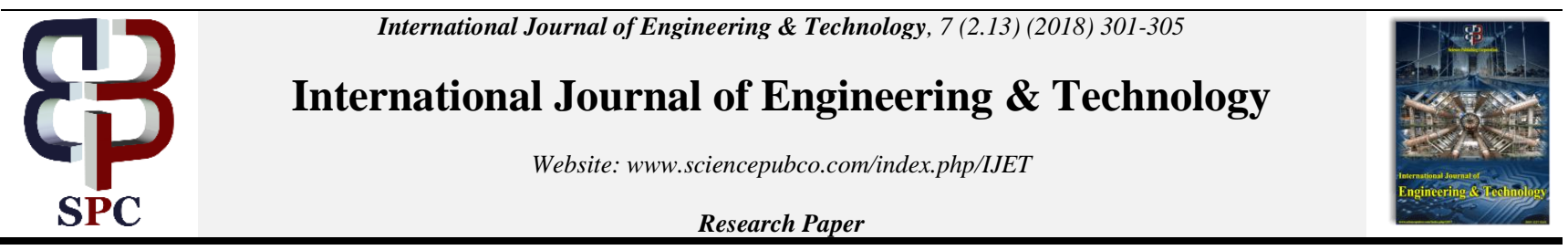

\title{
A Development Framework for Social Media Interaction in Learning Management System
}

\author{
Kalaivani Ravisekaran ${ }^{1 *}$, Sivakumar Ramakrishnan ${ }^{2}$ \\ ${ }^{1}$ Research Scholar, Department of Computer Science, \\ A.V.V.M. Sri Pushpam College(Autonomous) of Bharathidasan University, Thanjavur,India. \\ ${ }^{2}$ Associate Professor and Head, Department of Computer Science, \\ A.V.V.M. Sri Pushpam College (Autonomous), of Bharathidasan University, Thanjavur, India. \\ *Email: kalaivaniravisekaran@gmail.com
}

\begin{abstract}
Learning Management System (LMS) plays a vital role and an imprortant part in technology-enhanced learning. It presents different services to enhance the education procedure in ubiquitous learning condition. But, it is found that in existing education MS a extend range of education procedure and process are presented. But, it needs to be developed with some ubiquitous additional functionality. So the current research we proposed a struture for LMS to integrate social media services to make the LMS enrich in ubiquitous condition. This structure approves efficient social and intellectual interaction between users in ubiquitous Learning Management System environment.
\end{abstract}

Keywords: Ubiquitous computing, U- learning, Ubiquitous learning environments, Social Media, Social media tools, LMS

\section{Introduction}

Ubiquitous computing is currently emerging as a new computing paradigm shift where wireless and sensing technologies makes our daily life visually invisible [2]. With the advent of this new computing technology the learners in this learning environment progressed from traditional learning style to new learning style called "Ubiquitous learning named as (U-Learning)". It refers to a new learning standard, where learning happens at anywhere, anytime and at any device. This means [7]:

$>\quad$ Anywhere: Global accessible data's from various kinds of wireless communication networks and sensing technologies such as GPS, RFID tags, NFC, QR codes.

$>\quad$ Anytime: Access data $24 * 7$ and also independent of other services or persons.

$>\quad$ Any data: E-Mail, internet and intranet, public services and also allows the user's to access various data formats.

$>\quad$ Any device: Personal Digital Assistant (PDA), Tablet-PC, cell phone, RFID, NFC, smart gadgets, pads, smartphones etc.

The main characteristics of ubiquitous learning (U-Learning) are shown as follows:

- Permanency: The learner's data or information should be recorded continuously. Their data should never be missed unless it is purposefully deleted.

- Accessibility: The learner's data or information should be always available whenever the learners need to use it from anywhere.
- Immediacy: Wherever learners need their information's they can get any information immediately based on their requests.

The first approach is based on the transformation and review of the field of attitudes in the major intellectual domain of society. Based on this, the basic concepts of education are redefined. The need for rethinking in the intellectual domain of society is considered on the basis of a dynamic, changeable, and nonpersonal future. In this way, the macro education system changes the attitude of the student, teacher, content and school to the changes of time. Student and teacher look at the current education process from an inquiring, independent, creative and pluralistic perspective, and wants to move and create a learning-teaching-learning path by eliminating traditional and worn-out communication. From this point of view, concepts are inspired by contemporary social, political, and cultural philosophy, in accordance with the needs of a realistic and nonprejudicial society.

Allow learners to access personalized learning contents and enable knowledge reuse [4].

Due to the recent advancement and development of wireless and sensor technologies, information and communication system in learning environment, the Integration of ubiquitous learning with LMS has become a popular trend of education for the past few decades all over the world. This is because with Ulearning, learning can access their lectures, anytime, anywhere in the daily lives, regardless of their time, location and environment. It also has the ability to maximize the interaction among teachers, learners, parents and experts.

A ubiquitous learning environment is not only meant for common traditional classrooms. It should be applicable for any 
outside classroom spaces. The Lack of Social and intellectual interactions among learners in this interactive learning environment has become an important research gap in this new computing learning environment. By incorporating social media in LMS provides an easier and efficient way of communication among learners. It allows the users to easily share information among each other at anywhere, anytime and at any device.

Therefore, this paper presents a framework for social media interaction through ubiquitous learning environment in Learning Management System.

\section{Related Work}

Several approaches and works have been reported in the literature on the development and integration of ubiquitous features in Learning Management System.

Juan et.al [8] presented a new training model that uses social media integration into moodle using a plug-in Gleston. Next, Antonio Garmendia and Ruth cobos [10] proposed an extension of LMS with Social media services using the plug-in Social Media Format (SMF). Guozhen Zhang [9] developed a generalized social interaction support model for the ubiquitous learning. It support learners in increasing their social skills.

Subsequently, Evgeny et.al [11] developed an approach to extend the LMS with a plug-in enabling OpenSocial app to run with moodle. It allows Personal Learning Environment PLE functionalities to moodle.

Sarah Prestridge [12] came out with a conceptualization of using Twitter as a knowledge construction tool in mobile devices to investigate and increase the learning outcome of students. Another work reported by Claudia Megele [13] contributed a redesigned course module to enhance learners learning through integration of social media technologies into academic curriculum to access the learner's assessments.

Elvira Popescu [14] developed a platform eMUSE to provide collaborative learning support with social media in an integrated environment. Lockyer Lori and John Patterson [15] provides an education process with the integration of social networking technologies to enhance learners formal learning context process. It was specifically designed for formal learning context.
It is understood that from the literature survey, a wide range of effort have been taken in bringing LMS into ubiquitous learning environment. However, there exists some gaps, one such gap is to attain efficient intellectual interactions among teachers and learners in a technology-enhanced learning process. Hence this paper proposes a framework to integrate social media into LMS. The framework is demonstrated through Modular Object Oriented Developmental Learning Environment (MOODLE) and social media sites.

\section{A Framework for Social Media Integration Into LMS}

From this point of view, concepts are inspired by contemporary social, political, and cultural philosophy, in accordance with the needs of a realistic and non-prejudicial society.

The pivot, elite, and mythological education in education today has no place. Education work is teaching life. Therefore, to design the education system in line with the needs, or even today, we need something more complicated than a stereotypical and credulous system. In this way, the principle of democracy and participation, along with the institutionalization of thought as the task of a progressive and new system of education, is being considered. Obviously, institutionalizing requires the creation of a suitable platform for acceptance and tolerance with pluralistic views and thoughts and attitudes. In this way, the clashes are fierce and violent, rejected and unsuccessful. In this strategy, educational and evaluation methods are under revision. Educational and evaluation approaches are transformed from one-dimensional, quantitative, inefficient, flexible to flexible, efficient, and efficient ways. [1].

There are many approaches available to enable social media interaction in Learning Management System. But, they lack to provide efficient social and intellectual interaction among. Hence in this paper a development framework for integrating social media in Learning Management System is presented. The general framework view social media interaction is presented in Fig. 1. The activity diagram of students and course creators is shown in Fig.2.

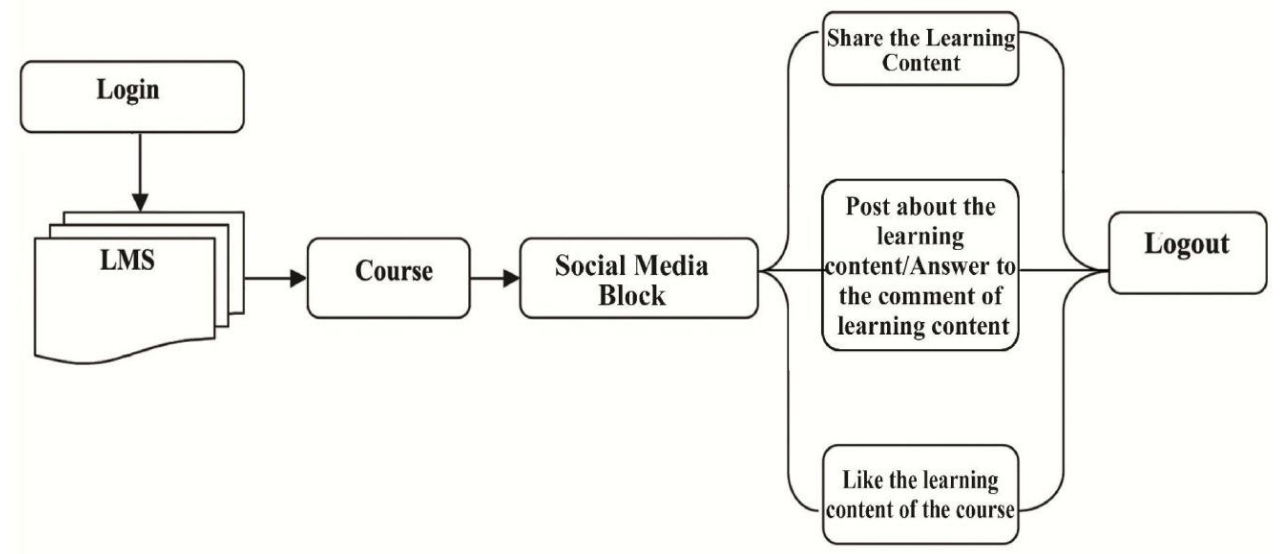

Fig. 1. General Framework for Social and Intellectual interaction. 

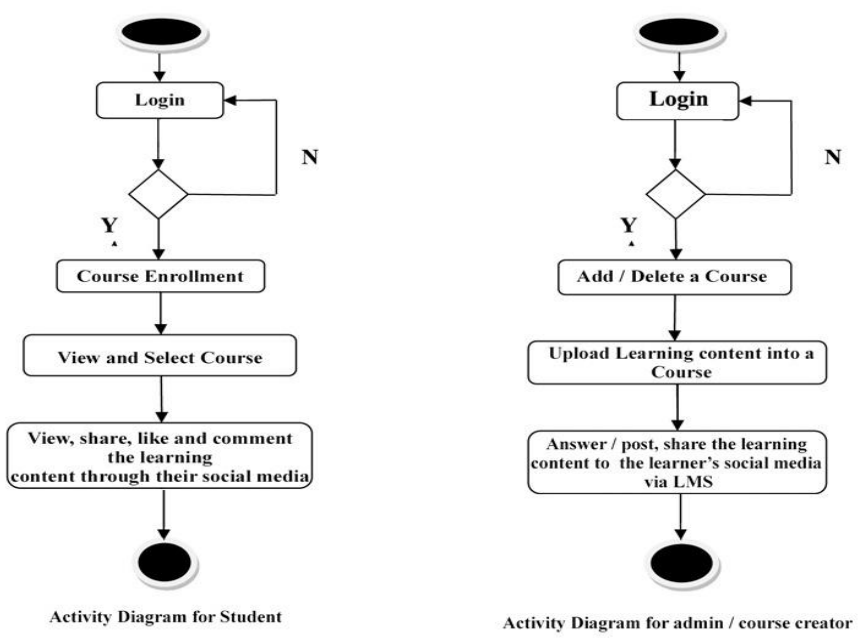

Fig.2. Activity diagram for Social and Intellectual interaction.

\section{Case study}

The developed structure is used in MOODLE software, a common LMS. It is found with LMS of A.V.V.M Sri Pushpam department, in India.

The LMS virtual education software and web conferencing have become the subject of computer technology and the use of the Internet, the topic of web conferencing or online meetings. As the LMS software manages and archives information and content and offline communication and the web conferencing system is used for online meetings and webinars, the combination of these two systems dramatically increases the performance and effectiveness of e-learning.

The LMS virtual learning software (LMS) and Web conference conferencing are consistent with the global development and synchronization with the technology of the day, in accordance with existing standards, is necessary and inevitable. On the other hand, the advent of information technology and the electronicization of information and its transfer in the world has led to the creation of the necessary fields and fields in areas such as virtual education and e-commerce, which has given a special meaning to the global village. This growing trend of the growing process leaves no room for the neglect of countries. The new information and communication technologies play an important role in teaching and learning, which, of course, are mentioned in many ways.

\section{Create a learner's course with Moodle:}

Click the Login Link.

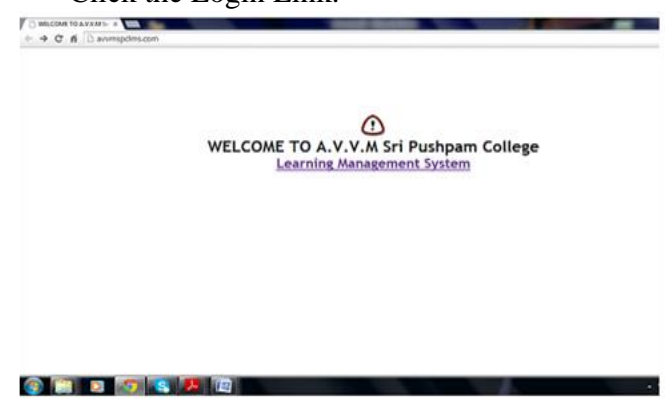

Fig.3. Login view

Create a new course.Go to settings- $>$ Site Administration$>$ Courses->Add/edit courses.course will be created. Select the course.

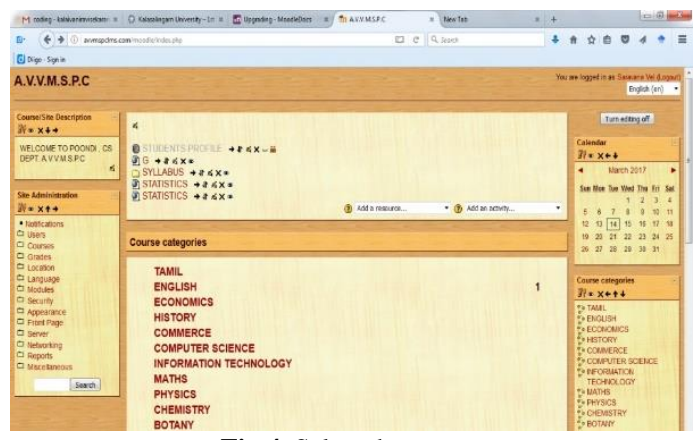

Fig.4. Select the course

Enrolled users:

Dashboard->my courses- $>$ any course name>participants->settings->enrolled users->Assign roles->enroll users.

To install social media tools Learning Management System(Moodle).The steps are as follows.

\section{Add a Block:}

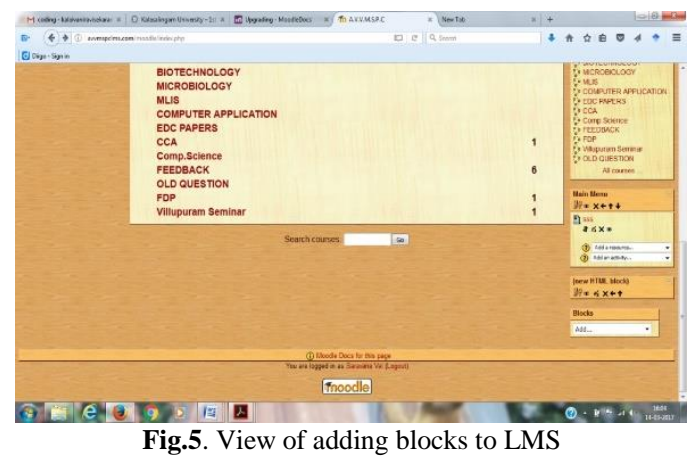

Click on block editing on->add a block ->choose HTML block. 


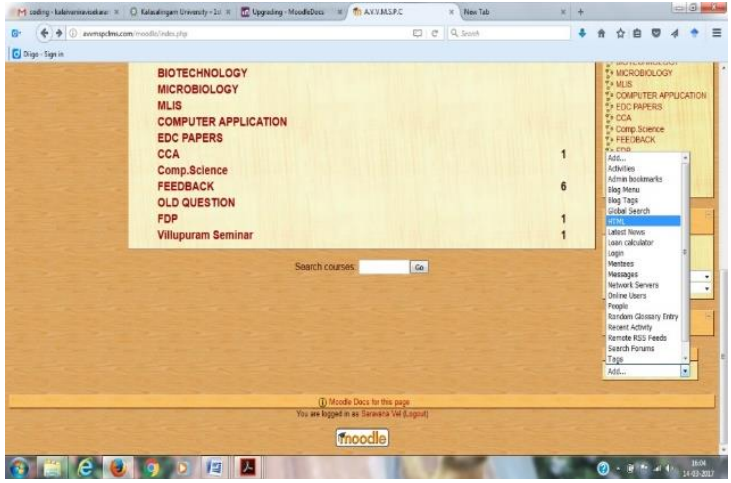

Fig.6. View of adding HTML code blocks to LMS

Create a social share tool:

Then click in configuring HTML block->block settings->block name->content->select the HTML code editor$>$ set the facebook,twitter and linkedIn codings.

\section{Facebook Button block codings:}

$<$ div class="fb-share-button" data-

href="https://developers.facebook.com/moodle spc/Moodlespc-1112175918908797/" data-layout="button_count" datamobile-iframe="true" $><$ a class="fb-xfbml-parse-ignore" target="_blank"href="https://www.facebook.com/sharer/sharer. php?u=https $\% 3 \mathrm{~A} \% 2 \mathrm{~F} \% 2 \mathrm{Fdevelopers.facebook.com} \% 2 \mathrm{Fmoodl}$ e+spc\&amp;src $=$ sdkpreparse" $>$ Share $\langle/ a\rangle\langle/$ div $\rangle$

\section{Twitter Button block coding:}

<a href="https://twitter.com/intent/tweet?@ moodleavvm1" class=" twitter-mention-button" =""="" data-showcount="false" $>$ Tweet $\langle/ \mathrm{a}\rangle$ $<$ script type="IN/Share" dataurl="http://localhost:8080/moodle" datacounter="right" $></$ script $>$

\section{LinkedIn Button block coding:}

<script src="//platform.linkedin.com/in.js" type="text/javascript" $>$ lang: en_US $</$ script $>$ $<$ script type="IN/Share" data-url="www.avvmspclms.com" data-counter="right" $>\langle/$ script $>$

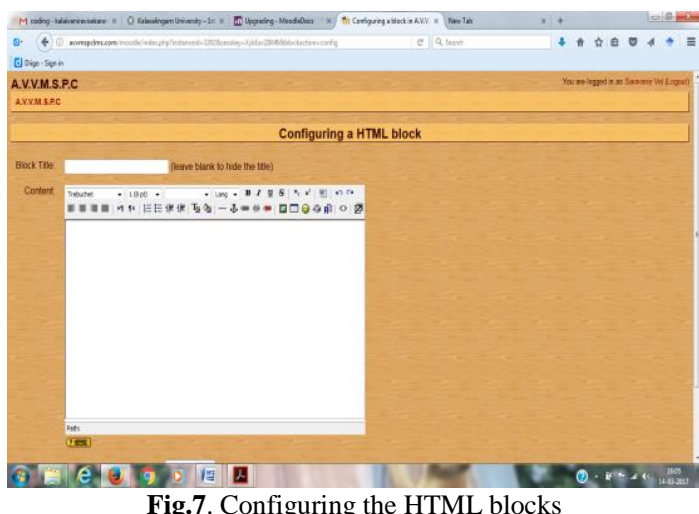

Fig.7. Configuring the HTML blocks

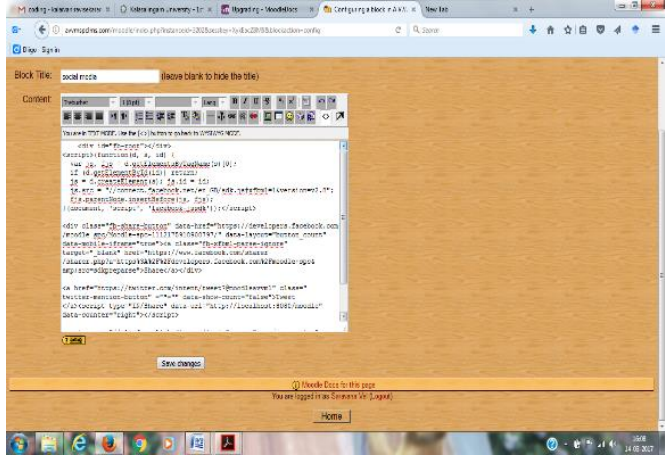

Fig.8. Source code for social media block

In the general settings, click the save changes button. To dispaly the social share block to appear on course page.

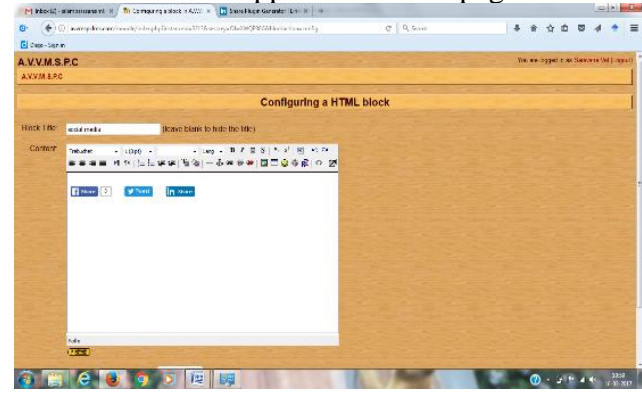

Fig.9. View of social media block configuration

\section{ADMIN VIEW:}

Click any social share button in social media block.open the moodle facebook,twitter or linkedIn page.share any content on moodle social page.

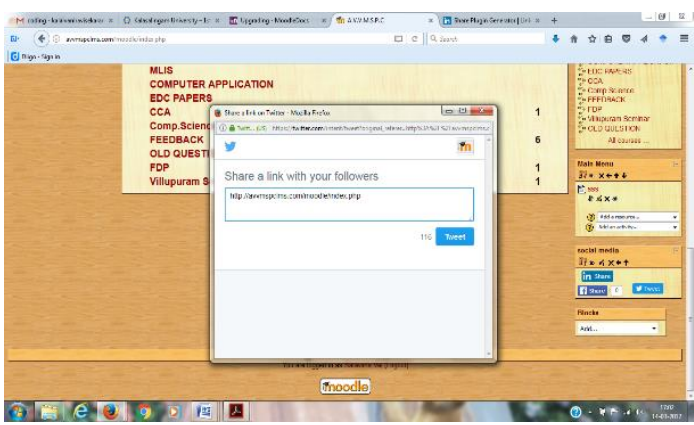

Fig.10. Tweeter social Media share View

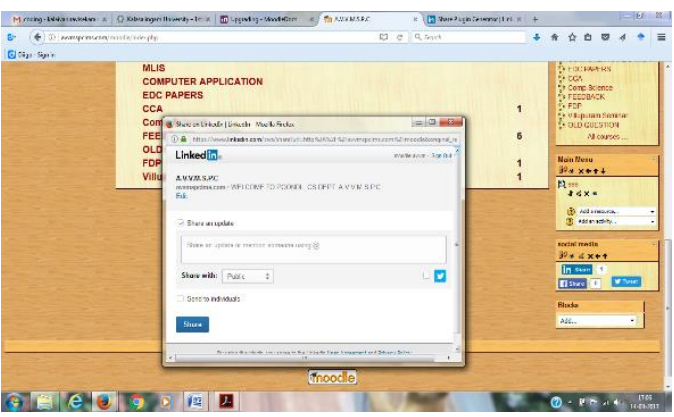

Fig.11. Linkedin social Media share View

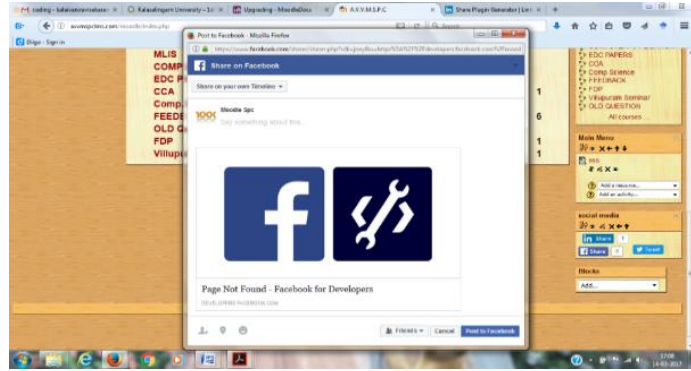

Fig.12. Facebook social Media share View 
Student view:

The learners can open there own facebook,twitter or LinkedIn account to view the notificationthen they can like,comment and share the learning content.

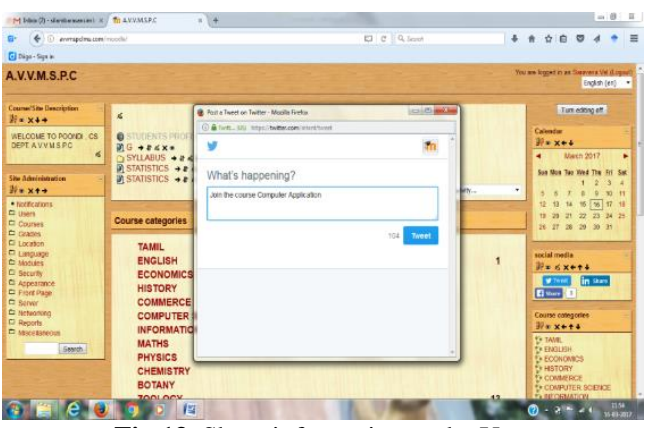

Fig.13. Share information to the User

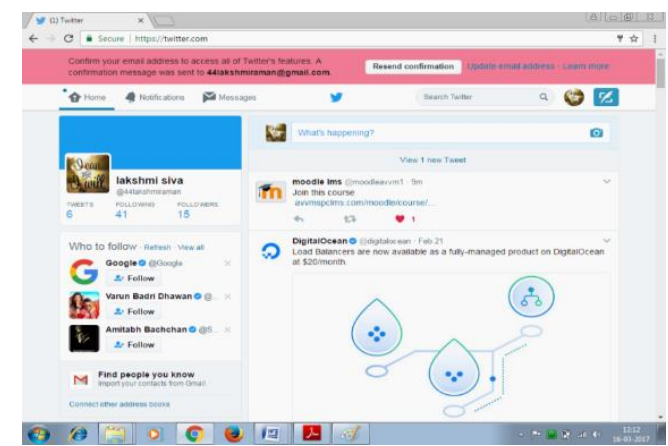

Fig.14. View of share information to the User in their social media

The framework was tested with a group of learners in the quesenery by participants.

\section{Conclusion}

A structure of combined social media in LMS were presented in this research. It is obviously found that by presenting a case study of educational institutions' LMS applying MOODLE. The major benefit of this method is to enable proper social and intellectual interactions among users, which approves the advantages of the efficiency of LMS in ubiquitous learning condition. The current paper can be developed with additional functionalities and enhancements of learners' behavior investigation. From this point of view, this article can be used to keep up the education of the country with the spirit of the times and the new approaches to education, and to meet the needs of the future generation and even the present generation along two paths, we can move on and consider two approaches.

\section{Reference}

[1] Sharma, A., and S. Vatta. "Role of learning management systems in education" International Journal of Advanced Research in Computer Science and Software Engineering 3 (2013): 6.

[2] Saadiah Yahya, Erny Arniza Ahmad and Kamarularifin Abd Jalil, "The definition and characteristics of ubiquitous learning: A discussion" International Journal of Education and Development using Information and Communication Technology (IJEDICT), 2010, Vol. 6, Issue 1, pp. 117-127.

[3] Shalabh Agarwal and Asoke Nath "Some Challenges in Designing and Implementing Learning Material for Ubiquitous E-learning Environment", Journal of Global Research in Computer Science,Vol. 2, February 2011.

[4]"Learning management system," http://edutechwiki.unige.ch/en/ Learning management_system.

[5]"LMS features," http://www.upsidelearning.com/learningmanagement-system-features.asp.

[6] "MOODLE," Available at https://moodle.org.

[7] G.J. Hwang, C.C. Tsai, and S.J. Yang, "Criteria, strategies and research issues of context-aware ubiquitous learning," Educational Technology \& Society, vol. 11(2), pp.81-91, 2008.

[8] J. Carlos, T. Diaz, et al. "Social learning environments." Interactive Collaborative Learning (ICL), 2015 International Conference on. IEEE, 2015.

[9] Zhang, Guozhen, Qun Jin, and Man Lin. "A framework of social interaction support for ubiquitous learning." Advanced Information Networking and Applications, 2005. AINA 2005. 19th International Conference on. Vol. 2. IEEE, 2005.

[10] Garmendía, Antonio, and Ruth Cobos. "Towards the extension of a LMS with Social Media services." International Conference on Cooperative Design, Visualization and Engineering. Springer, Berlin, Heidelberg, 2013.

[11] Bogdanov, Evgeny, et al. "From LMS to PLE: A step forward through opensocial apps in moodle." Advances in Web-Based Learning-ICWL 2012 (2012): 69-78.

[12] Prestridge, Sarah. "A focus on students' use of Twitter-their interactions with each other, content and interface." Active Learning in Higher Education 15.2 (2014): 101-115.

[13] Fakoor, M., Kosari, A., Jafarzadeh, M. Revision on fuzzy artificial potential field for humanoid robot path planning in unknown environment, International Journal of Advanced Mechatronic Systems, Volume 6, Issue 4, page 174-183, 2015.

[14] Fakoor, M., Kosari, A., Jafarzadeh, M. Journal of Applied Research and Technology, Journal of Applied Research and Technology, Volume 14, Issue 5, 2016, 300-310, 2016.

[15] Lori, and J. Patterson. "Integrating social networking technologies in education: a case study of a formal learning environment." Advanced Learning Technologies, 2008. ICALT'08. Eighth IEEE International Conference on. IEEE, 2008. 